\title{
FLORA MARINA BENTÓNICA DE LA REGIÓN AUSTRAL DE SUDAMÉRICA Y LA ANTÁRTICA
}

\author{
BENTHIC MARINE FLORA FROM SOUTHERN SOUTH AMERICA AND ANTARCTICA
}

\author{
María Eliana Ramírez ${ }^{1}$
}

\begin{abstract}
RESUMEN
El conocimiento taxonómico de la flora marina bentónica de la región austral de Sudamérica, incluida la Antártica, está basado fundamentalmente en los registros de las expediciones europeas que recorrieron los mares australes durante los siglos XVIII, XIX y principios del siglo XX. Debido a la lejanía de estos territorios y a las dificultades logísticas para la realización de nuevas recolecciones en el área, poco se ha avanzado en la revisión crítica de los taxa publicados en la literatura hasta la fecha. El presente trabajo da a conocer un listado actualizado de esta flora marina, en base a las publicaciones y exploraciones recientes en el área. Adicionalmente y en base a este listado, se presenta un análisis biogeográfico con el objeto de establecer las relaciones entre la flora marina de la región austral de Sudamérica y aquélla de la región antártica.

Palabras clave: Chile, algas marinas, biogeografía
\end{abstract}

\section{ABSTRACT}

Knowledge of the benthic marine flora from southern South America, and Antarctica is based on past centuries of exploration of the region by early naturalists. Lack of accessibility to the area has been a limiting factor in carrying out a critical revision of these early records reported in the literature.

The aim of the present work is to update the benthic marine algal records for the area. In addition, a preliminary assessment is made of the biogeographic relationships between the marine flora of southern South America and the Antarctic region.

Key words: Chile, marine algae, biogeography.

\section{INTRODUCCIÓN}

El conocimiento taxonómico actual de la flora marina de la región austral de Sudamérica, incluida la Antártica, es el resultado de las exploraciones realizadas por expedicionarios y naturalistas extranjeros durante los siglos XVIII, XIX y principios del siglo $\mathrm{XX}$. Gran parte de esta información se encuentra dispersa en la literatura, en forma de listados de especies determinadas por los más reconocidos ficólogos de la época, tales como: C. A Agardh, Jacob Agardh, F. Kutzing, C. Montagne, P. Hariot y C. Skottsberg, entre otros.

El material estudiado, incluido el material tipo de muchas especies descritas como nuevas para la ciencia, quedó depositado en los principales museos europeos como el de Londres (BC), de París, en Francia (PC), de Leiden en Holanda y los Museos de Lund ( LD) y Goteborg (GT) en Suecia. Las dificultades para revisar el material tipo de muchas especies, así como la documentación de estas primeras colecciones, ha sido un factor limitante en el estudio y revisión crítica de estos taxa, algunos de los cuales presentan una confusa historia taxonómica y nomenclatural.

Los trabajos más destacados relativos a las algas marinas bentónicas de la zona austral de 
Sudamérica, están incluidos en las obras de Hooker \& Harvey (1845), Hooker (1847), Hariot (1889), Svedelius (1900), Hylmö (1919, 1938), Cotton (1915), Skottsberg (1907, 1921, 1923, 1941 ), Kylin \& Skottsberg (1919) y Lemoine (1920), entre otros. Una recopilación de la bibliografía y distribución de las algas de estas regiones se encuentra en los Catálogos de Papenfuss (1964), Ramírez (1982) y Ramírez \& Santelices (1991). Con posterioridad a estas expediciones y a la publicación de los correspondientes trabajos, las investigaciones sobre esta flora, en especial la del área de Magallanes - Tierra del Fuego y de las islas Malvinas o Falkland, han sido esporádicos y relativamente escasos. Entre ellos, se puede mencionar a Searles (1978), Mendoza \& Cabioch (1985), Ramírez \& Peters (1992), Fredericq et al. (1992), Mendoza et al. (1996), Peters et al. (2000), Peters \& Ramírez (2001) y Kim et al. (2004).

Por el contrario, el conocimiento taxonómico de la flora marina de la Antártica e islas subantárticas adyacentes, se ha incrementado sostenidamente en los últimos 50 años, destacándose entre éstos a: Asensi (1966), Delepine (1967), Zaneveld 1968, Moe \& DeLaca (1976), Moe \& Silva (1979,1980, 1983,1989), Moe (1985, 1986a , 1986b), Lamb \& Zimmermann (1977), Zielinski (1981), Wynne (1982), Ricker (1987), Cormaci et al. (1992), Muller et al. (1992), John et al. (1994a, 1994b), Fredericq \& Ramírez (1996), Clayton et al. (1997), Gallardo et al. (1999), Alongi et al. (2002), Wiencke \& Clayton (2002), Le Gall et al. (2008) y Hommersand et al. (2009), entre otros.

Se conoce que la Antártica estuvo unida al extremo austral de Sudamérica desde el Mesozoico y que su separación habría ocurrido sólo en los últimos 30 millones de años (Crame 1992, Clayton 1994). Dicha separación aisló totalmente a la península Antártica de Sudamérica, con la consecuente evolución de una flora marina propia, adaptada a las aguas frías. La pregunta es si a causa de esta separación, ¿existe aún alguna relación entre la flora marina de las regiones subantárticas de Sudamérica con sus correspondientes antárticos y cuál sería esta exacta relación?

El presente trabajo entrega un registro actualizado de las especies de macroalgas presentes en las regiones de Magallanes - Tierra del Fuego, islas Malvinas o Falkland y la región antárctica con sus respectivas distribuciones geográficas, con el propósito de evaluar de manera preliminar la biogeografía del área.

\section{MATERIAL Y MÉTODOS}

El listado actualizado de los taxa se realizó sobre la base de los trabajos publicados, y a registros de herbarios provenientes de nuevas colecciones realizadas por la autora en el área de Magallanes, Tierra del Fuego e islas Malvinas-Falkland, las que se encuentran debidamente documentadas en la base de datos pertenecientes al Herbario Nacional (SGO), del Museo Nacional de Historia Natural de Chile.

El análisis biogeográfico de esta flora se realizó sobre la base de este listado aplicando la metodología de Sokal \& Sneath (1963) de los "pares ponderados", previo cálculo del índice de similitud de Jaccard, utilizando para ello los valores "1" para presencia de la especie en la localidad y "0" ausencia de la especie en la localidad.

\section{RESULTADOS}

En la actualidad, y basado en esta nueva lista de especies, la flora marina bentónica de las islas Malvinas-Falkland comprende un total de 169 especies; la flora de Magallanes y Tierra del Fuego 234 especies y la flora marina de la Antártica 115 especies (Tabla 1). El análisis de similitud florística entre las tres localidades se presenta en la figura 1 , en la forma de un dendrograma. Los resultados indican que existen dos grupos separados, uno formado por Magallanes-Tierra del Fuego y las islas Malvinas-Falkland, y el otro por la Antártica. La similitud florística en el primer grupo es de un $60 \%$, y la relación de este grupo con la Antártica es de un $23 \%$. Esto indica que la región antártica se separa del grupo subantártico de Sudamérica austral como un ensamble separado. 
TABLA 1. Lista de especies de algas marinas bentónicas por localidad. El número 1 indica presencia y 0 ausencia.

\begin{tabular}{ccc}
\hline Especies & Islas Malvinas- \\
& Falkland & $\begin{array}{c}\text { Magallanes y } \\
\text { Fierra del } \\
\text { Fuego }\end{array}$
\end{tabular} Antártica

División Chlorophyta

Acrosiphonia arcta (Dillwyn) J. Ag.

Acrosiphonia pacifica (Montagne ) J. Ag.

Blidingia minima (Nageli ex Kutzing)

\begin{tabular}{|c|c|}
\hline 0 & 0 \\
\hline 1 & 1 \\
\hline 0 & 1 \\
\hline 1 & 1 \\
\hline 0 & 1 \\
\hline 1 & 1 \\
\hline 0 & 1 \\
\hline 0 & 1 \\
\hline 0 & 1 \\
\hline 1 & 1 \\
\hline 1 & 1 \\
\hline 1 & 1 \\
\hline 0 & 1 \\
\hline 1 & 0 \\
\hline 1 & 1 \\
\hline 0 & 1 \\
\hline 0 & 0 \\
\hline 0 & 1 \\
\hline 1 & 1 \\
\hline 0 & 1 \\
\hline 0 & 1 \\
\hline 1 & 1 \\
\hline 1 & 1 \\
\hline 0 & 1 \\
\hline 1 & 1 \\
\hline 0 & 1 \\
\hline 0 & 0 \\
\hline 0 & 0 \\
\hline 1 & 1 \\
\hline 0 & 1 \\
\hline 1 & 0 \\
\hline
\end{tabular}

Bryopsis australis Sonder

\begin{tabular}{|c|c|}
\hline 0 & 0 \\
\hline 1 & 1 \\
\hline 0 & 1 \\
\hline 1 & 1 \\
\hline 0 & 1 \\
\hline 1 & 1 \\
\hline 0 & 1 \\
\hline 0 & 1 \\
\hline 0 & 1 \\
\hline 1 & 1 \\
\hline 1 & 1 \\
\hline 1 & 1 \\
\hline 0 & 1 \\
\hline 1 & 0 \\
\hline 1 & 1 \\
\hline 0 & 1 \\
\hline 0 & 0 \\
\hline 0 & 1 \\
\hline 1 & 1 \\
\hline 0 & 1 \\
\hline 0 & 1 \\
\hline 1 & 1 \\
\hline 1 & 1 \\
\hline 0 & 1 \\
\hline 1 & 1 \\
\hline 0 & 1 \\
\hline 0 & 0 \\
\hline 0 & 0 \\
\hline 1 & 1 \\
\hline 0 & 1 \\
\hline 1 & 0 \\
\hline
\end{tabular}

Bryopsis magellanica Hylmo

1

Bryopsis rosae Gaudichaud

Chaetomorpha dubyana Kutzing

Cladophora anisogona (Mont.) Kutzing

Cladophora australis Rabenhorst

Cladophora confusa Hariot

Cladophora falklandica Hook. \& Harvey

Cladophora flexuosa Hariot

Cladophora glauscescens (Griffith) Hook. \& Harv.

Cladophora gracilis (Griffiths) Kutzing

Cladophora incompta (Hook.\& Harv.) Hook.

Cladophora magellanica Ardissone

Cladophora repens (J. Ag.) Harvey

Cladophora rupestris (Linnaeus) Kutz.

Cladophora subsimplex Kutzing

Cladophoropsis brachyartrus (Svedelius) Borgesen

Cladophoropsis voluticola (Hariot) Borgesen

Codium difforme? Kutzing

Codium fragile (Suringar) Hariot

Codium subantarcticum Silva sp. Ined.

Entocladia maculans (Cotton) Papenfuss

Gomontia arrhiza Hariot

Lambia antarctica (Skottsberg) Delepine

Lola irregularis Zaneveld

Monostroma fuscum (Post. \& Ruprecht) Wittrock.

Monostroma grevillei (Thuret) Wittrock

Monostroma hariotii Gain

\section{1} 1 1 0 0 0 0 0 0 0 0 0 0 0 0 0 1 0 0 0 0 0 0 0 1 0 1 1 0 0 1 
Ostreobium quecketii Bornet \& Flahaut

\begin{tabular}{|c|c|}
\hline 1 & 1 \\
\hline 0 & 1 \\
\hline 1 & 1 \\
\hline 0 & 1 \\
\hline 1 & 1 \\
\hline 1 & 1 \\
\hline 1 & 1 \\
\hline 0 & 1 \\
\hline 0 & 0 \\
\hline 0 & 1 \\
\hline 0 & 1 \\
\hline 1 & 1 \\
\hline 1 & 1 \\
\hline 1 & 1 \\
\hline 1 & 1 \\
\hline 0 & 1 \\
\hline 1 & 1 \\
\hline 0 & 1 \\
\hline 1 & 1 \\
\hline
\end{tabular}

Percusaria percursa (J. Agardh )Rosenvinge

Prasiola crispa (Lightfoot) Kutzing. forma Ant-

Prasiola tesellata (Hook. \& Harv.) Kutz. 0

Rhizoclonium ambiguum (Hook. \& Harv.) Kutz.

Rhizoclonium capillare? (Kutz.) Hariot

Rhizoclonium riparium (Roth) Harvey

Rhizoclonium tortuosum (Dillwyn) Kutz.

Ulothrix australis Gain

Ulothrix flaca (Dillwyn) Thuret

Ulothrix implexa Kutzing

0

Ulva lactuca Linnaeus

Ulva bulbosa (Sur) Hariot

Ulva chlatrata (Roth) C. Agardh

Ulva compressa Linnaeus

Ulva intestinalis Linnaeus

Ulva linza Linnaeus 0

Urospora penicilliformis (Roth) Areschough

División Ochrophyta/Chrysophyceae

Antarctosaccion applanatum (Gain) Delep. 0

Vaucheria patagonica Hylmo

Adenocystis utricularis (Bory) Skottsberg

Adenocystis utricularis forma longissima Skottsberg

Ascoseira mirabilis Skottsberg

Alethocladus corymbosus (Dickie) Sauvageau

Chordaria linearis (Hook f. \& Harv.) Cotton 1

Chordaria magellanica Kylin $\quad 1$

Cladochroa chnoosporiformis Skottsberg 1

Cladostephus spongiosus (Hudson) C. Agardh 1

Cladothele decaisne Hooker \& Harvey 1

Corycus lanceolatus (Kjellman) Kutzing $\quad 1$

Cystosphaera jacquinotii (Montagne) Skottsberg 0

Desmarestia anceps Montagne

0

1

$1 \quad 0$

1

0

$\begin{array}{lll}0 & 1\end{array}$


Desmarestia antarctica Moe \& Silva 0

Desmarestia chordalis Hooker \& Harvey 1

Desmarestia confervoides (Bory) Ramírez \& AF

Desmarestia distans (C. Agardh) J.Agardh $\quad 1$

Desmarestia ligulata (Stackh.) Lamouroux

Desmarestia menziesii J. Agardh

0

Desmarestia mullerii Ramírez \& Peters

Dictyosiphon hirsutus (Skottsberg) Pedersen

Durvillaea antarctica (Chamissoi) Hariot

Durvillaea harveyii? Hooker

Ectocarpus fasciculatus Harvey

Ectocarpus siliculosus (Dillwyn) Lyngbye

Elachista antarctica Skottsberg

Geminocarpus geminatus (Hook. \& Harv.) Skottsberg

Geminocarpus austrogeorgiae Skottsberg

1

1

1

1

1

1

1

1

0

1

0

1

0

Gononema pectinatum (Skottsb.) Kuck. \& Skottsberg

1

Gononema ramosum (Skottsb.) Kuck. \& Skottsberg

Halopteris funicularis (Montagne) Sauvageau

1

Halopteris obovata (Hook. \& Harv.) Sauvageau

Hecatonema maculans (Collins) Sauvageau

Himantothallus grandifolius (A \& E. Gepp) Zinova

Leathesia difformis (Linnaeus) Areschough

Leptonematella falklandica (Skottsberg) Wynne

Lessonia flavicans Bory

1

1

0

0

0

1

1

Lessonia nigrescens Bory

0

Lessonia vadosa Searles

Lessonia searlesiana Asensi \& De Reviers

Lithoderma piliferum Skottsberg

Lithoderma antarcticum Skottsberg

0

Macrocystis pyrifera (Linnaeus) J. Agardh

1

Myriactula pusilla (Skottsberg) Skottsberg

Myriactula rosarioides (Skottsberg) Skottsberg

Myrionema densum Skottsberg

Myrionema fueguianum Skottsberg

0

Myrionema incommodum Skottsberg 
Myrionema macrocarpum Skottsberg

Myrionema patagonicum Skottsberg

Myrionema vulgare Thuret

Petalonia fascia ( O.F. Muller) Kuntze

Petroderma maculiforme (Wollny) Kuckuck

Phaerus antarcticus Skottsberg

Punctaria plantaginea (Roth) Greville

Pylaiella littoralis (Linnaeus) Kjellman

Ralfsia australis Skottsberg

Scytosiphon lomentaria (Lyngbye) Link

Scytothamnus australis (J. Agardh) Hook. \& Harv.

Scytothamnus fasciculatus (Hook. \& Harv.) Cotton

Sphacelaria bornetii Hariot

Sphacelaria cirrosa (Roth) Greville

Sphacelaria furcigera Kutzing

Spongonema tomentosum (Hudson) Kutzing

Stereocladon rugulosus (Bory) Hariot

Streblonema patagonicum Skottsberg

Syringoderma australe Levring

Tinocladia falklandica (Skottsberg) Kylin

Utriculidium durvillei Skottsberg

1

1

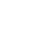

0

1

1

1

1

1

1

0

0

1

0

1

0

0

1

Division Rhodophyta

Acanthococcus antarcticus Hookker f. \& Harvey

Acrochaetium catenulatum Howe

Acrochaetium fueguensis Kylin

Acrochaetium leuringii Papenfuss

0

Acrochaetium macropus Kylin

Ahnfeltia plicata (Hudson) EM Fries

Amphiroa crassa Lamouroux

Amphiroa cretacea Postels \& Ruprecht

Amphiroa tasmanica Sonder

Antarcticothamnion polysporum Moe \& Silva

Antartocolax lambii Skottsberg

Antithamnion plumula (Ellis) Thuret

$\begin{array}{lll}1 & 1 & 0 \\ 0 & 1 & 0 \\ 0 & 1 & 0 \\ 1 & 1 & 0 \\ 1 & 1 & 0 \\ 0 & 1 & 1 \\ 0 & 1 & 0 \\ 0 & 1 & 0 \\ 0 & 1 & 0 \\ 0 & 0 & 1 \\ 0 & 0 & 1 \\ 0 & 1 & 0\end{array}$


Antithamnion ptilota (Hooker \& Harvey) Harvey- $\quad 0$

Antithamnionella sarniensis Lyle 0

Antithamnionella ternifolia (Hooker \& Harvey) Lyle $\quad 0$

Audouinella concrescens (K.M.Drew) Dixon 0

Audouinella membranaceae (Magnus) Papenfuss

Audouinella purpurea (Lightfoot) Woelkerling

Austropugetia crassa Moe

Ballia callitricha (C. Agardh) Kutzing

Ballia sertularioides (Suhr) Papenfuss

Bangia atropurpurea (Roth) C. Agardh

Callithamnion gaudichaudii C. Agardh

Calithamnion leptocladum Montagne

Callithamnion montagnei J. D. Hooker

Calithamnion spinuliferum Ardissone

Calithamnion subsecudum Grunow

Callophyllis atrosanguinea (Hooker f.

Callophyllis fastigiata (J. Agardh) J. Agardh

Callophyllis multifida (Reinsch) Kylin

Callophyllis tenera J.Agardh

Callophyllis variegata (Bory) Kutzing

Camontagnea oxyclada Pujals

Catenella fusiformis (C. Agardh) Skottsberg

Ceramium circinatum (Kutzing) C. Agardh

Ceramium diaphanum (Lightfoot) Roth

Ceramium dozei Hariot

Ceramium involutum Kutzing

1

(1)

1

0

1

0

0

1

0

0

1

0

1

0

0

1

1

0

1

1

0

1

0

1

1

Ceramium rubrum C. Agardh

Ceramium strictum Roth

Ceramium ungulatum (Kutzing) Hariot

Chondria macrocarpa Harvey

Cladodonta lyalii (Hooker \& Harvey) Skottsberg

Clathromorphum obtectulum (Foslie) WH Adey

Clathromorphum lemoineanum Mendoza \& Cabioch

Colacodasya inconspicua (Reinsch)

Schmitz \& Falkenberg 
Colaconema membranaceum (Magnus) Woelkerling

0

Colacopsis lophurellae Kylin

1

Corallina elongata Ellis \& Solander

1

Corallina officinalis var. chilensis

( Decaisne) Kutzing

Corallina pilulifera Postels \& Ruprecht

Curdiaea racovitzae Hariot

"Cystoclonium" obtusangulum Kutzing

Dasyptilon Harveyii (Hooker f.) Papenfuss

Delesseria epiglossum Skottsberg

Delesseria fueguensis Skottsberg

Delesseria lancifolia (Hooker f.) J. Agardh

Delesseria macloviana Skottsberg

Delesseria salicifolia Reinsch

Delesseria stephanocarpa

Delisea pulcra (Greville) Montagne

Epymenia falklandica Taylor

Epymenia obtusa (Greville) Kutzing

Erythrotrichia carnea (Dilwyn) J. Agardh

Fosliella farinosa (Lamouroux) Howe

Gainia mollis RL Moe

Gelidium crinale (Turner) Gaillon

Gelidium lingulatum Kutzing

Georgiella confluens (Reinsch) Kylin

Gigartina skottsbergii Setchell \& Gardner

Glaphyrosiphon chilensis Ramírez, Leister

\& Gabrielson (ex Grateloupia intestinalis)

Gracilaria?pulvinata Skottsberg

Grateloupia doryophora (Montagne) Howe

Grateloupia filicina? (Lamouroux) C. Agardh

Griffithsia antarctica Hooker f. \& Harvey

Gymnogongrus antarcticus Skottsberg

Gymnogongrus turquetii Hariot

Herposiphonia sullivanae (Hooker \& Harvey)

Falkenberg

Heterosiphonia berkeleyii Montagne

Heterosiphonia punicea (Montagne) Kylin

Hildenbrandia lecanellieri Hariot

1

1

0

1

1

1

0

1

1

1

0

0

1

0

0

0

0

1

0

1

1

0

1

1

0

1

1

0

1

1

1

1

Hydrolithon discoideum (Foslie) Mendoza \& Cabioch

1

1

Hydrolithon subantarcticum (Foslie)

ML Mendoza \& Cabioch

0

1

1

1

0

1

0

1

1

0

1

1

1

1

110

$\begin{array}{lll}1 & 0\end{array}$

0

1

1

1

10

$1 \quad 0$

$\begin{array}{lll}1 & 0\end{array}$

$\begin{array}{lll}1 & 1\end{array}$

$0 \quad 1$

$\begin{array}{lll}0 & 1\end{array}$

1

$\begin{array}{ll}1 & 0\end{array}$

110

$\begin{array}{lll}1 & 1\end{array}$

$\begin{array}{ll}1 & 0\end{array}$

$\begin{array}{lll}1 & 1\end{array}$ 
Hymenena durvillaei (Bory) Kylin

Hymenena falklandica (Kylin)

0

1

Hymenena laciniata (Hooker \& Harvey) Kylin

Hymenocladiopsis prolifera (Reinsch) Wynne

Iridaea cordata (Turner) Bory

Iridaea mawsonii AHS Lucas

Iridaea tuberculosa ( Hooker \& Harvey) Leister

Laurencia chilensis de Toni, Forti

Leniea lubrica Moe

Leptophytum coulmanicum (Foslie) WH Adey

Lithophyllum almanense Lemoine

Lithophyllum atalayense Lemoine

Lithophyllum decussatum

(Ellis \& Solander) Filipos

Lithophyllum pustulatum (Lamouroux) Foslie

Lithophyllum racemus (Lamouroux) Foslie

Lithothamnion caroli Lemoine

Lithothamnion granuliferum Foslie

Lithothamnion heterocladum Foslie

Lithothamnion müelleri Lenormand ex Rosanoff

Lithothamnion pauciporosum Lemoine

Lithothamnion rugosum Foslie

Lithothamnion validum Foslie

Lophurella comosa (Hooker \& Harvey)

Falkenberg

Lophurella hookeriana (J. Agardh) Falkenberg

Lophurella patula (Hooker \& Harvey) de Toni

Mazzaella laminarioides (Bory) Fredericq

Mazzaella membranacea (J. Agardh) Fredericq

Medeiothamnion flaccidum (Hooker \& Harvey)

Brauner

Meiodiscus concrescens (K.M. Drew) Gabrielson

Mesophyllum fuegianum (Foslie) Adey

Microrhinus carnosus (Reinsch) Skottsberg

Mychodea carnosa Hooker \& Harvey

Mychodea compressa Harvey

Myriogramme crozierii? ( Hooker \& Harvey) Kylin

Myriogramme livida (Hooker \& Harvey) Kylin

Myriogramme manginii (Gain) Skottsberg

Myriogramme smithii (Hooker\& Harvey) Kylin

Neogoniolithon mamillare (Harvey) Setchell \& Albañil

Nereoginkgo adiantifolia Kylin
1

0

1

0

1

0

0

0

0

0

0

0

0

0

1

1

0

0

1

0

1

1

1

1

0

1

0

1

0

0

0

0

1

0

1

0

0
0

0

0

1

1

1

0

0

1

1

0

0

0

0

0

0

1

1

0

0

0

0

0

0

0

0

0

0

1

0

1

0

0

0

1

1

1

0

1 
Nereoginkgo populifolia Moe

Nitophyllum paessleri Pilger

Nothogenia fastigiata (Bory) Parkinson

Notophycus fimbriatus RL Moe

Pachymenia orbicularis (Zanardini) Setchell \& Gardner

Palmaria decipiens (Reinsch) Ricker

Pantoneura plocamioides Kylin

Phycodrys antarctica (Skottsberg) Skottsberg

Phycodrys austrogeorgica Skottsberg

Phycodrys quercifolia (Bory) Skottsberg

Phyllophora antarctica A \& E Gepp

Phyllophora ahnfeltioides Skottsberg

Phymatolithon foecundum (Kjellman)

Duwel \& Wegeberg

Phymatolithon lenormandii Areschough) Adey

Picconiella pectinata( Hooker \& Harvey)

de Toni fil.

Piconiella plumosa (Kylin) J. De Toni

Platyclinia fueguensis Skottsberg

Platyclinia taylorii Levring

Plocamium cartilagineum (Linnaeus) Dixon

Plocamium hookerii Harvey

Plocamium secundatum ( Kutzing) Kutzing

Plumariopsis peninsularis Moe \& Silva

Polycoryne radiata Skottsberg

Polysiphonia abscissa Hooker \& Harvey

Polysiphonia anisogona Hooker \& Harvey

Polysiphonia corymbifera (C. Agardh) Endlicher

Polysiphonia hasslerii Taylor

Polysiphonia morrowi Harvey

Polysiphonia tenuistriata Hooker \& Harvey

Polysiphonia urceolata

(Lightfoot ex Dillwyn) Greville

Polysiphonia virgata (C. Agardh) Sprengel

Porphyra columbina Montagne

Porphyra cuneiformis

(Setchell \& Hus) Krishnamurthy

Porphyra endiviifolia (A. \& E. Gepp)

Chamberlain

Porphyra plocamiestris RW Ricker

Porphyra umbilicalis Kutzing

Porphyra woolhousiae Harvey

Pseudolaingia larsenii (Skottsberg) Levring

Pseudolithophyllum fuegianum ( Heydrich)

Mendoza \& Cabioch
0

$\begin{array}{lll}0 & 0 & 1 \\ 0 & 1 & 0 \\ 1 & 1 & 0 \\ 0 & 0 & 1 \\ 0 & 0 & 1\end{array}$

0

0

0

0

1

0

0

0

$\mathrm{O}$

0

0

0

0

0

1

1

0

1

1

1

0

0

0

0

0

0

1

0

0

0

1

1

1

1 
Pseudophycodrys phyllophora (J. Agardh)

Skottsberg

Pterosiphonia pennata (C. Agardh) Falkenberg

Pterothamnion antarcticum (Kylin) Moe \& Silva

Pterothamnion simile (Hooker \& Harvey) Nageli

Ptilonia magellanica (Montagne) J. Agardh

Rhodochorton purpureum (Lightfoot)

Rosenvinge

Rhodokrambe laingioides Moe

Rhodophyllis centrocarpa (Montagne) Wynne

Rhodymenia flabellifolia? (Bory) Montagne

Rhodymenia palmatiformis Skottsberg

Rhodymenia subantarctica RW Ricker

Sarcothalia circumcincta (J.Agardh) Hommersand

Sarcothalia crispata ( Bory) Leister

Sarcothalia dichotoma

(Hooker \& Harvey) Leister

Sarcothalia papillosa (Bory) Leister

Schizoseris condensata (Reinsch) Ricker

Schizoseris dichotoma (Hooker \& Harvey) Kylin

Schizoseris laciniata (Kutzing) Kylin

Schyzymenia binderii (J. Agardh) J. Agardh

Sporoglossum lophurellae Kylin

Stictosiphonia hookerii Hooker

Synarthrophyton patena

(Hooker f. \& Harvey) RA Towsend

Synarthrophyton schmitzii (Hariot) Mendoza, Molina \& Ventura

Trematocarpus antarticus (Hariot) Fredericq

Varimenia macropustulosa Moe

\begin{tabular}{|c|c|}
\hline & 1 \\
\hline 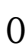 & 1 \\
\hline & 0 \\
\hline & 0 \\
\hline & 1 \\
\hline & 0 \\
\hline & 0 \\
\hline & 1 \\
\hline & 1 \\
\hline & 1 \\
\hline & 1 \\
\hline & 0 \\
\hline & 1 \\
\hline & 1 \\
\hline & 1 \\
\hline & 1 \\
\hline & 1 \\
\hline & 1 \\
\hline & 1 \\
\hline & 1 \\
\hline & 1 \\
\hline & 1 \\
\hline & 1 \\
\hline & 0 \\
\hline & 0 \\
\hline
\end{tabular}

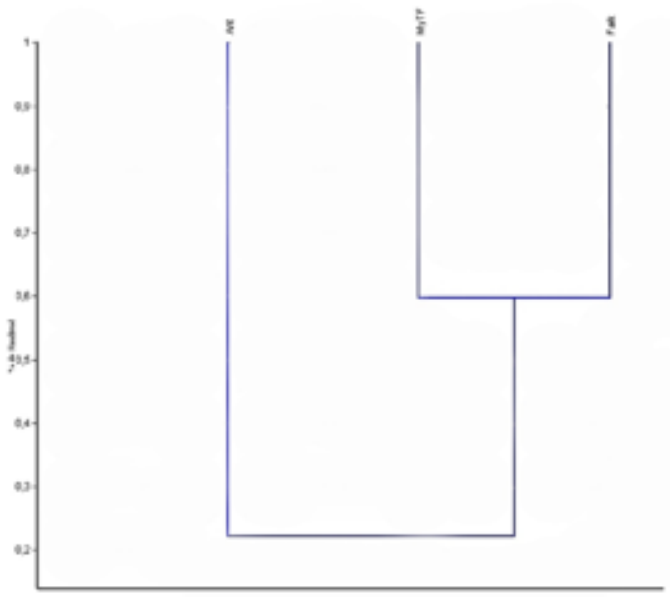

Fig.1. Dendrograma que muestra los valores de similitud entre la flora marina de la región austral de Sudamérica, Magallanes-Tierra del Fuego (MyTF) con las islas Malvinas-Falkland (Falk.), y de éstas con Antártica (Ant.). 


\section{DISCUSIÓN Y CONCLUSIONES}

De los datos obtenidos se puede concluir que, de las tres localidades consideradas en este análisis (islas Malvinas o Falkland, Magallanes-Tierra del Fuego, y Antártica), la región de Magallanes-Tierra del Fuego es la que, comparativamente, presenta el mayor número de especies ( 234 especies, versus 169 en las islas Malvinas y 115 en la Antártica).

Aunque, en términos de superficie territorial, las distintas áreas no pueden ser comparadas para efectos de conocer la diversidad de macroalgas, lo que sí es relevante es la disponibilidad de sustratos y otras condiciones ecológicas tales como luz y temperatura requeridas para el asentamiento y sobrevida de estos organismos. Por ejemplo, cabe citar la situación de la Antártica, con una extensa superficie territorial, donde gran parte de sus costas presentan hábitats discontinuos, con escasos sustratos estables y condiciones extremas de temperatura que son limitantes para el asentamiento de los organismos bentónicos; sólo la península Antártica, las islas Shetland del Sur y algunas islas del este de la Antártica, permiten la existencia y sobrevida de las macroalgas. Consecuentemente, los registros de la flora marina bentónica de la Antártica provienen sólo de estos lugares.

La figura 1 muestra que las localidades de Malvinas-Falkland y Magallanes-Tierra del Fuego están relacionadas, presentando un valor de similitud de $60 \%$; y que este conjunto se relaciona con el grupo de la región antártica en un porcentaje de similitud menor (23\%). Lo que muestra el dendrograma, en consecuencia, son dos ensambles separados, uno formado por Malvinas y Magallanes-Tierra del Fuego, y el otro por la Antártica. Estos resultados no son congruentes con lo señalado por John et al. (1994b), quienes consideran que más que una separación entre las floras de la región antártica y de las regiones subantárticas adyacentes del extremo austral de Sudamérica, existiría una continuidad entre estas floras, admitiendo la idea de la existencia de una sola y gran provincia biogeográfica. Tampoco estos resultados son coincidentes con lo propuesto por Clarke \& Crame (1989) para la fauna marina de aguas someras, quienes encontraron una alta similitud entre algunos grupos de invertebrados marinos de la región antártica y del área subantártica de Sudamérica, atribuyendo esto a la relativamente temprana separación entre Sudamérica y la península Antártica (30 millones de años). Clarke \& Crame
(1989) postulan, además, que incluso después del aislamiento de las aguas antárticas, las islas del Mar de Scotia pueden haber servido como ruta de dispersión para estos animales entre Sudamérica y la Antártica.

Hommersand et al. (2009), basados en datos moleculares de un conjunto de algas rojas no publicados a la fecha (secuenciación del gene rbcL), afirman que la relación entre algunos géneros y especies de algas rojas de la península Antártica con aquéllas de Sudamérica es compleja, con algunos grupos de especies muy distantes filogenéticamente, como lo que ocurre con las algas rojas de Nueva Zelanda y las de la Antártica, y con otros grupos más cercanos como ocurre con especies de algas rojas presentes en Nueva Zelanda y en Sudáfrica. Hommersand et al. (2009) argumentan que existe la posibilidad que las singularidades del clima antártico haya causado una evolución más rápida del gene rbcL en la Antártica que en aguas templadas del sur de Sudamérica, hecho que debe ser determinado a través de nuevos estudios. Los resultados del presente trabajo estarían de acuerdo con lo postulado por Hommersand et al. (2009), ya que para la totalidad de las especies de la flora marina bentónica presentes en la región antártica y en las áreas subantárticas adyacentes de Sudamérica austral, existe un porcentaje de similitud de sólo $23 \%$. De acuerdo con Hommersand et al. (2009), la Antártica, las islas Shetland del Sur, las Orcadas del Sur y Georgia del Sur, conforman definitivamente una provincia biogeográfica, por incluir géneros y especies que están distantes de los elementos florísticos presentes en Nueva Zelanda y Tasmania, Sudáfrica y Namibia, el sur de Sudamérica, las islas Malvinas y otras islas subantárticas como las islas Crozet, Kerguelen, Macquarie, Cambell y Auckland. Por otra parte, el alto porcentaje de similitud (60\%), encontrado entre la flora marina de las islas Malvinas con la flora de Magallanes y Tierra del Fuego, es consecuencia del número de especies de origen subantártico compartido entre ambas áreas, lo que es atribuible a la dispersión de esta flora a través de la corriente circumpolar antártica o West Wind Drift (WWD).

Se recomienda profundizar los estudios taxonómicos de estas localidades a un nivel que supere la taxonomía alfa para tener una idea más comprensiva y una hipótesis más certera sobre la relación de las flora marina de la región antártica y la del extremo austral de Sudamérica.. 


\section{LITERATURA CITADA}

Alongi, G., M. Cormaci \& G. Furnari 2002. The Corallinaceae (Rhodophyta) from the Ross Sea (Antarctica): a taxonomic revision rejects all records except Phymatolithon foecundum. Phycologia 41: 140-146.

Asensi, A. O. La presencia de Pylaiella littoralis (L.) Kjellm en la Antártica. Contribución del Instituto Antártico Argentino 101: 14 pp.

Clarke, A \& J.A. Crame 1989. The origin of the Southern Ocean marine fauna. In Crame, J.A. (Ed.). Origin and Evolution of the Antarctic Biota. The Geological Society, London, pp.253-268.

Clayton, M. 1994. Evolution of the Antarctic marine benthic Algal flora. J. Phycol. 30: 897-904.

Clayton, M.N., C. Wiencke \& H. Kloser 1997. New records of temperate and subantarctic marine Benthic macroalgae from Antarctica. Polar Biology 17:141-149.

Cormaci, M., G. Furnari \& B. Scammacca 1992. The benthic algal flora of Terra Nova Bay (Ross Sea, Antarctica). Bot. Mar. 35:541-552.

Cotton, A. D. 1915. Cryptogams from the Falkland Islands collected by Mrs. Vallentin and described by A.D. Cotton. Journal Linnean Society, Botany 43:137-231, pl. 4-10.

Crame, J. A. 1992. Evolutionary history of the Polar regions. Hist.Biol. 6:37-60.

Delépine, R. 1967. Sur un nouveau genre de Chlorophycées antarctiques, Lambia. Compte rendu hebdomadaire des séances de l'Académie des Sciences, París 264:1410-1413.

Fredericq, S., M. H. Hommersand \& G.L. Leister 1992. Morphology and systematics of Acanthococcus antarcticus ( Cystocloniaceae, Hodophyta). Phycologia 31:101-118.

Fredericq, S. \& M.E. Ramírez 1996. Systematic studies of the Antarctic species of the Phyllophoraceae (Gigartinales, Rhodophyta) based on $\mathrm{rbcL}$ sequence analysis. Hydrobiologia 326/327:137-143.

Gallardo, T., I. M. Pérez-Ruzafa, A. Flores-Moya \& F. Conde 1999. New collections of Benthic marine algae from Livingston and Deception Islands (South Shetland Islands) and Trinity Island (Bransfield Strait). Botanica Marina 42:61-69.
Hariot, P. 1889. Algues. Mission Scientifique du Cape Horn, 1882-1883. Botanique 5:1-109. Hommersand, M. H., R. L. Moe, C. D. Amsler \& S. Fredericq 2009. Notes on the systematics and biogeographical relantionships of Antarctic and sub-antarctic Rhodophyta with descriptions of four new genera and five new species. Botanica Marina 52:509-534.

Hooker, J. D. 1847. The Botany of the Antarctic Voyage of H.M. Discovery ships Erebus and Terror in the years 1839-1843. I. Flora Antarctica. Part 2. Botany of Fuegia, The Falkland, Kerguelen's Land, etc. London (1845-1847). Algae: pp. 454-502, pls.165-194.

Hooker, J. D. \& W. H. Harvey 1845. Algae Antarcticae... London. Journ. Bot. 4:249-276, 293-298.

Hylmö, D. 1919. Zur kenntnis der subatarktischen. In Nordensjold, O. (ed.). Wissenchafliche Ergebnisse der Schwedischen SudpolarExpedition 1901-1903, 4(4):1-20.

Hylmö, D. 1938. Botanische Ergebnisse der Schwedischen Expedition nach Patagonien und dem Feurlande 1907-1909. XI. Meeresalgen 3. Chlorophyceae. Kongliga Svenkabers Vetenskabs Akademiens Hadlingar ser.3. 17 (1):1-23.

John, D., P. Pugh \& I. Tittley 1994a. Observations on the benthic marine algal flora of South Georgia: a floristic and ecological analysis: Bull. Br. Mus. Nat. Hist. (Bot.).24(2): 101-114.

John, D., I. Tittley, W. Lawson \& P. Pugh 1994b. Distribution of seaweeds floras in the Southern Oceans. Bot. Mar. 37:235-239.

Kim, M., E. C. Yang \& A. Mansilla 2004. Recent introduction of Polysiphonia morrowii ( Ceramiales, Rhodophyta) to Punta Arenas, Chile. Bot. Mar. 47:389-394.

Kylin, H. \& C. Skottsberg 1919. Zur kenntnis der Subantarktischen und Antarktischen Meeresalgen II. Rhodophyceen. In Nordensjold, O. (ed). Wissenschaftliche Ergebnisse der Swedischen Sudpolar Expedition 1901-1903 4: 2(15):1-88.

Lamb, I. \& M. Zimmermann 1977. Benthic marine algae of the Antarctic Peninsula. Antarctic Res. Ser. Washington 23:129-229.

Le Gall, L., J. L. Dalen \& G. W. Saunders 2008. Phylogenetic analyses of the red algal order 
Rhodymeniales supports recognition of the Hymenocladiaceae fam. nov., Fryeellaceae fam.nov., and Neogastroclonium gen.nov. J. Phycol. 44:1556-1571.

Lemoine, P. 1920. Botanische Ergebnisse der Swedischen Expedition nach Patagonien und der Feurlande 1907-1909. VII. Les Melobesiees. Kongliga Svenska Vetenskaps Akademiens Handlingar 61 (4):1-17.

Mendoza, M. L. \& J. Cabioch 1985. Critique et comparaison morphogenétique des genres Clathromorphum et Antarcticophyllum (Rhodophyta, Corallinaceae). Conséquences biogéographyques et systématiques . Cah. Biol. Mar. 26:251-266.

Mendoza, M. L., S. Molina \& P. Valenzuela 1996. In: Flora Criptogámica de Tierra del Fuego. Rhodophyta: Corallinales. Tomo VIII- Fascículo 3: Guarrera, De Amos \& Matteri (eds.): 72 pp.

Moe, R. 1985. Gainia and Gainiaceae, a new genus and family of crustose marine Rhodophyceae from Antarctica. Phycology 24:419-428.

Moe, R. 1986a. Notophycus fimbriatus (Solieraceae), a new genus and species of marine Rhodophyceae from the Antarctic Peninsula. Phycologia 25: 544-550.

Moe, R. 1986b. Hymenocladiopsis crustiginea (Rhodymeniaceae). A new genus and species of marine Rhodophyceae from the Antarctic Peninsula. Phycology 25: 1-9.

Moe, R. \& T. E. DeLaca 1976. Ocurrence of macroscopic algae along the Antarctic Peninsula. Antarctic Journal 11:20-24.

Moe, R. \& P. Silva 1979. Morphological and taxonomic studies on Antarctic Ceramiaceae (Rhodophyceae).I. Antarcticothamnion polysporum gen. Et sp. Nov. British Phycological Journal 14:385-405.

Moe, R. \& P. Silva 1980. Morphological and taxonomic studies on Antarctic Ceramiaceae (Rhodophyceae). II. Pterothamnion antarcticum (Kylin) comb.nov. (Antithamnion antarcticum Kylin). British Phycological Journal 15: 1-17.

Moe, R \& P. Silva 1983. Morphological and taxonomic studies on Antarctic Ceramiaceae (Rhodophyceae) III. Georgiella and Plumariopsis.(Tribe Ptiloteae). British Phycological Journal 18:275-298.

Moe, R. \& P. Silva 1989. Desmarestia antarctica (Desmarestiales, Phaeophyceae) A new ligulate species with an endophytic gametophyte. $\mathrm{Pl}$. Syst. Evol. 164: 273-283.

Muller, D. G., M. E. Ramírez \& R.Westermeir 1992. Utriculidium durvillei (Bory?) Skottsberg en isla Rey Jorge, Antártica. Serie Científica INACH 42:47-52.

Papenfuss, G. 1964. Catalogue and Bibliography of Antarctic and subantarctic benthic marine algae. Am. Geophys. Union, Antarctic Res. Ser., 1:1-76.

Peters, A. F., M. E. Ramírez \& A. Rulke 2000. The phylogenetic position of the subantarctic marine macroalga Desmarestia chordalis (Phaeophyceae) inferred from nuclear ribosomal ITS sequences. Pol. Biol. 23:95-99.

Peters, A. F. \& M. E. Ramírez 2001. Molecular phylogeny of small brown algae, with special reference to the systematic position of Caepidium antarcticum (Adenocystaceae, Ectocarpales). Crypt. Algol. 22(2):187-200.

Ramírez, M.E. 1982. Catálogo de las algas marinas del Territorio Chileno Antártico. Serie Científica INACH 29:39-67.

Ramírez, M. E. \& B. Santelices 1991. Catálogo de las algas marinas bentónicas de la Costa del Pacifico Temperado de Sudamérica. Monografías Biológicas 5. Pontificia Universidad Católica de Chile. Santiago, Chile. 433 pp.

Ramirez, M. E. \& A. F. Peters 1992. The South American species of Desmarestia (Phaeophyceae) . Can. J. of Bot. 70:2430-2445.

Ricker, R. 1987. Taxonomy and Biogeography of Macquarie Islands Seaweeds. British Museum Natural History. London: 344 pp.

Searles, R. B. 1978. The genus Lessonia Bory (Phaeophyta, Laminariales) in Southern Chile and Argentina. Brit. Phycol. J. 13: 361-381.

Skottsberg, C. 1907. Zur kenntnis der Subantarktischen und Antarktischen meeresalgen I. Phaeophyceen. In: Nordensjold O. (ed). Wissenschaftliche Ergebnisse der Schwedischen Sudpolar Expedition 1901-1903. 4 (6): 1-172.

Skottsberg, C. 1921. Marine Algae I. Phaeophyceae. In: Botanische Ergebnisse der Schwedischen Expedition nach Patagonien und der Feurlande 1907-1909. VII. Kongliga Svenska Vetenskap Akademiens Handlingar 61 (11) :1-56.

Skottsberg, C. 1923. Marine Algae 2. Rhodophyceae. In: Botanische Ergebnisse der Schwedischen Expedition nach Patagonien und der Feurlande 
1907-1909. Kongliga Svenska Vetenskaps Akademiens Handlingar 63 (8):1-70.

Skottsberg, C. 1941. Communities of marine algae in subantarctic and Antarctic waters. Kongliga Suenska Vetenskap Akademiens Handlingar, Tredje Serien 19(4):1-92, 3 plates.

Sokal, R \& P.H. Sneath 1963. Principles of numerical taxonomy. W.H. Freeman and Company, San Francisco. $359 \mathrm{pp}$.

Svedelius, N. 1900. Algen aus den Landern der Magellanstrasse und West Patagonien I. Chlorophyceae. In : Nordensjold O. (ed). Wissenschaftliche Ergebnisse der Schwedischen Expedition nach Magellanslandern 1895-1897. 3:283-316.

Wiencke, C. \& M. N. Clayton 2002. Antarctic seaweeds.
In:(J. W. Wagele, ed.). Synopsis of the antarctic benthos, A.R.G. Gantner Verlag KG . Ruggell/ Lichtensteien. 159 pp., 40 pls.

Wynne, M. J. 1982. Observations on four species of Delesseriaceae (Rhodophyta) from the South Sandwich Islands, The Antarctic. Contr. Univ. Mich. Herb. 15:325-337.

Zaneveld, J.S. 1968. Benthic marine algae, Ross Island to Balleny Islands. Antarctic Map Folio Series. American Geographycal Society New York, Folio 10:1-12, 13 plates.

Zielinski, K. 1990. Benthic macroalgae of Admirality Bay (King George Island, South Shetlands, Antarctica). Polish Polar Research 2:71-94. 